\title{
Electron spin torque in atoms
}

\section{$\operatorname{AUTHOR}(\mathrm{S})$ :}

Hara, Takaaki; Senami, Masato; Tachibana, Akitomo

\section{CITATION:}

Hara, Takaaki ...[et al]. Electron spin torque in atoms. Physics Letters A 2012, 376(17): 1434-1441

ISSUE DATE:

2012-03

URL:

http://hdl.handle.net/2433/155089

\section{RIGHT:}

C 2012 Elsevier B.V.; この論文は出版社版でありません。引用の際には 出版社版をご確認ご利用ください。; This is not the published version. Please cite only the published version. 


\title{
Electron spin torque in atoms
}

Takaaki Hara ${ }^{\mathrm{a}}$, Masato Senami ${ }^{\mathrm{a}}$, Akitomo Tachibana ${ }^{\mathrm{a}}$

${ }^{a}$ Department of Micro Engineering, Kyoto University, Kyoto 606-8501, Japan

\begin{abstract}
The spin torque and zeta force, which govern spin dynamics, are studied by using monoatoms in their steady states. We find nonzero local spin torque in transition metal atoms, which is in balance with the counter torque, the zeta force. We show that d-orbital electrons have a crucial effect on these torques. Nonzero local chirality density in transition metal atoms is also found, though the electron mass has the effect to wash out nonzero chirality density. Distribution patterns of the chirality density are the same for Sc-Ni atoms, though the electron density distributions are different.
\end{abstract}

\section{Introduction}

Recently, electron spin has received considerable attention due to the great development of spintronics [1]. One of representative examples is a disk read-and-write head [2], which brought in today's high capacity hard disk drives. The usage of spin is expected to achieve a further breakthrough in the field of information processing and storage. Almost all spintronics devices realize many functions by controlling electron spin e.g. due to magnetic fields. Therefore, the efficient control of the spin is an essential key for spintronics devices. In fact, spin-polarized current injection and a magnetic field are often used to control magnetization vectors. For example, spin transfer torque RAM, which is now widely studied, uses the transfer of spin-aligned electrons.

For the control of the spin, the torque for the spin is the most important quantity. Nowadays, spin-transfer torque has widely been researched as the torque affecting the spin [3-5]. This torque corresponds to the transfer of spin angular momentum from spin-polarized current to a magnetic element. This can be given as the form, $-\frac{\hbar}{2 e}\left(\vec{j}_{s} \cdot \nabla\right) \vec{n}$, where $\vec{j}_{s}$ denotes spin current density and $\vec{n}$ is a magnetization vector. As the force for moving domain wall, another spin torque perpendicular to the above torque is also studied $[6-8] ; \beta \vec{m} \times(\vec{j} \cdot \nabla) \vec{m}$, where $\vec{j}$ is current density. However, most of these approaches are based on phenomenological grounds, such as analyses with the Landau-Lifshitz-Gilbert equation and the exchange interaction between spin-polarized current and localized spin.

In this work, we propose a generic and essential description of the torque for the spin [9-11], which is derived from first principles. Hence, the essential property of the spin dynamics can be studied by our approach. In addition, phenomenological spin torques as mentioned above

Email address: akitomo@scl.kyoto-u.ac.jp (Akitomo Tachibana) are explained in a microscopic point of view. In this paper, we pick up various atoms of main group elements and transition metals and investigate the steady state of electron spin. Surprisingly, we find that the steady state of spin in atoms, particularly transition metals, is realized in a balance between nonzero local spin torque and its counter torque, the zeta force. In addition, we show that the magnitude of the spin torque is significantly dependent on electrons in d-orbitals.

In this work, our purpose is the establishment of the validity of the spin torque and zeta force and the investigation of properties of them. Hence, our scope is restricted to the steady states of monoatoms. After we are familiar enough with these novel quantities, we will clarify popular phenomenological torques for electronic spin and the dynamics of any spin states from electron wave functions.

\section{Theory and calculation methods}

\subsection{Spin torque and zeta force}

The quantities of the spin torque and the zeta force are proposed by Tachibana $[9,10]$. These quantities clarify the dynamics of spin. The degree of freedom of the spin can naturally be described in the relativistic theory. In the Dirac equation, the spin degree of freedom is intrinsically included. As explained later, the spin torque and the zeta force are derived only for the four-component electronic states in the relativistic theory. Hence, throughout this study, our discussion is restricted to the relativistic theory.

Before the definitions of the spin torque and zeta force are introduced, we consider the spin angular momentum density operator in quantum field theory [10],

$$
\frac{1}{2} \hbar \hat{\sigma}_{e}^{k}(\vec{r})=\hat{\psi}^{\dagger}(\vec{r}) \frac{1}{2} \hbar \Sigma^{k} \hat{\psi}(\vec{r})
$$

where $k=1-3$ and $\hat{\psi}$ is the four-component spinor. 
The dynamics of electron spin is defined by the time evolution of the spin angular momentum density operator. Hence, the equation of motion of the spin angular momentum density is given by

$$
\frac{\partial}{\partial t}\left[\frac{\hbar}{2} \hat{\sigma}_{e}^{k}(\vec{r})\right]=\hat{t}_{e}^{k}(\vec{r})+\hat{\zeta}_{e}^{k}(\vec{r}) .
$$

The time derivative of the spin angular momentum is reduced by using the ordinary Dirac equation. We can classify the right-hand side into two parts, the spin torque term $\hat{t}_{e}^{k}(\vec{r})$ and the zeta force term $\hat{\zeta}_{e}^{k}(\vec{r})$. The first term corresponds to the ordinary spin torque term, which comes from the Heisenberg equation in quantum mechanics, $d \vec{\sigma} / d t=-(i / \hbar)[\vec{\sigma}, H]$. The latter zeta force, which is chiral force, appears in quantum field theory. The expectation value of the zeta force for finite system vanishes, since the integration over the whole region is zero. The spin torque is related to the relativistic stress tensor density, and the zeta force is rewritten by using the chirality density. These are considered to originate in e.g., spinorbit and spin-spin interactions, and magnetic fields. At the steady state of the spin, the spin torque should be in balance with the zeta force.

The definition of the spin torque density operator is given by

$$
\hat{t}_{e}^{k}(\vec{r})=-\epsilon_{\operatorname{lnk}} \hat{\tau}_{e}^{\Pi l n}(\vec{r}),
$$

where $\epsilon_{l n k}$ is the Levi-Civita tensor and $\hat{\tau}_{e}^{\Pi l n}(\vec{r})$ is the relativistic stress tensor density operator,

$$
\hat{\tau}_{e}^{\Pi k l}(\vec{r})=\frac{i \hbar c}{2}\left[\hat{\bar{\psi}}(\vec{r}) \gamma^{l} \hat{D}_{k}(\vec{r}) \hat{\psi}(\vec{r})-\hat{D}_{k}^{\dagger}(\vec{r}) \hat{\bar{\psi}}(\vec{r}) \gamma^{l} \hat{\psi}(\vec{r})\right]
$$

Here, $c$ is the speed of light in the vacuum and $\hat{D}_{k}$ is the covariant derivative which is given by the derivative operator and the vector potential $\hat{A}_{k}$,

$$
\hat{D}_{k}(\vec{r})=\partial_{k}-i \frac{Z_{e} e}{\hbar c} \hat{A}^{k}(\vec{r}) .
$$

Here $Z_{e}=-1$ is for the electron. The stress tensor density is known to classify properties of chemical bonding [12-20]. The relativistic stress tensor (4) is a Hermite operator, and however not symmetric tensor for the exchange of indexes $k$ and $l$. On the other hand, the nonrelativistic stress tensor is symmetric, and hence the spin torque arises from the asymmetric property due to relativistic effects, as mentioned above. Hence, to see this relativistic effect, we must use a relativistic four-component wave function. As shown in Eqs. (3) - (5), the definition of the spin torque includes the vector potential. Hence, the spin torque is the driving force of the rotational motion of spin induced by a magnetic field.

The other term is the zeta force,

$$
\hat{\zeta}_{e}^{k}(\vec{r})=-c \partial_{k}\left[\hat{\bar{\psi}}(\vec{r}) \gamma^{k} \frac{1}{2} \hbar \Sigma^{k} \hat{\psi}(\vec{r})\right],
$$

where the multiple appearance of the index $k$ does not mean the summation over $k$ in this equation. Equation (6) is reduced to

$$
\hat{\zeta}_{e}^{k}(\vec{r})=\frac{\hbar}{2 e} \partial_{k} \hat{j}_{5}^{0}(\vec{r})
$$

where $\hat{j}_{5}^{0}$ is the zeroth component of the chiral current density operator $\hat{j}_{5}^{\mu}(\mu=0-3)$,

$$
\begin{aligned}
\hat{j}_{5}^{\mu}(\vec{r}) & =-c e\left[\hat{\bar{\psi}}(\vec{r}) \gamma^{\mu} \gamma_{5} \hat{\psi}(\vec{r})\right], \\
\gamma_{5} & =i \gamma^{0} \gamma^{1} \gamma^{2} \gamma^{3} .
\end{aligned}
$$

As you can see after easy algebra, the zeta force and the spin torque are derived as a product of the large component $\left(\psi_{1}\right.$ and $\psi_{2}$ in the Dirac representation) and the small one $\left(\psi_{3}\right.$ and $\psi_{4}$ in the Dirac representation) of a four-component spinor. Therefore, it can be concluded again that the spin torque and the zeta force are quantities arising from the relativistic effects.

The zeroth component of the chiral current is the chirality density [21]. The meaning of the chirality density is more apparent in the following expression,

$$
\hat{j}_{5}^{0}(\vec{r})=-c e\left[\hat{\psi}_{R}^{\dagger} \hat{\psi}_{R}-\hat{\psi}_{L}^{\dagger} \hat{\psi}_{L}\right]
$$

where, $\hat{\psi}_{L}$ and $\hat{\psi}_{R}$ are the left-handed and right-handed spinors. These operators are defined as

$$
\hat{\psi}_{L}=\hat{P}_{L} \psi, \quad \hat{\psi}_{R}=\hat{P}_{R} \psi
$$

where $\hat{P}_{L}$ and $\hat{P}_{R}$ are chirality projection operators,

$$
\hat{P}_{L} \equiv \frac{1-\gamma_{5}}{2}, \quad \hat{P}_{R} \equiv \frac{1+\gamma_{5}}{2}
$$

The left-handed and right-handed electrons are flipped to each other by the mass term. In the following, we will see this for free electrons. Since $\gamma_{5} \gamma^{\mu}+\gamma^{\mu} \gamma_{5}=0$, equations for $\hat{\psi}$ and $\gamma_{5} \hat{\psi}$ are derived as

$$
\begin{aligned}
& \left(i \gamma^{\mu} \partial_{\mu}-m\right) \psi=0, \\
& \left(i \gamma^{\mu} \partial_{\mu}+m\right) \gamma_{5} \psi=0 .
\end{aligned}
$$

By these two equations and Eq. (12),

$$
\begin{aligned}
i \gamma^{\mu} \partial_{\mu} \hat{\psi}_{R} & =m \hat{\psi}_{L} \\
i \gamma^{\mu} \partial_{\mu} \hat{\psi}_{L} & =m \hat{\psi}_{R}
\end{aligned}
$$

For massless electron, $m=0$, Eqs. (15) and (16) are independent of each other. Otherwise, $\hat{\psi}_{L}$ and $\hat{\psi}_{R}$ are mixed due to their Dirac mass term. Hence, the chirality density is not conserved, unless an electron has much larger kinetic energy than the electrons mass. We will show in the next section that the chirality density in atoms actually generates zeta force and controls the dynamics of electron spin. 
Table 1: Computational details of CI.

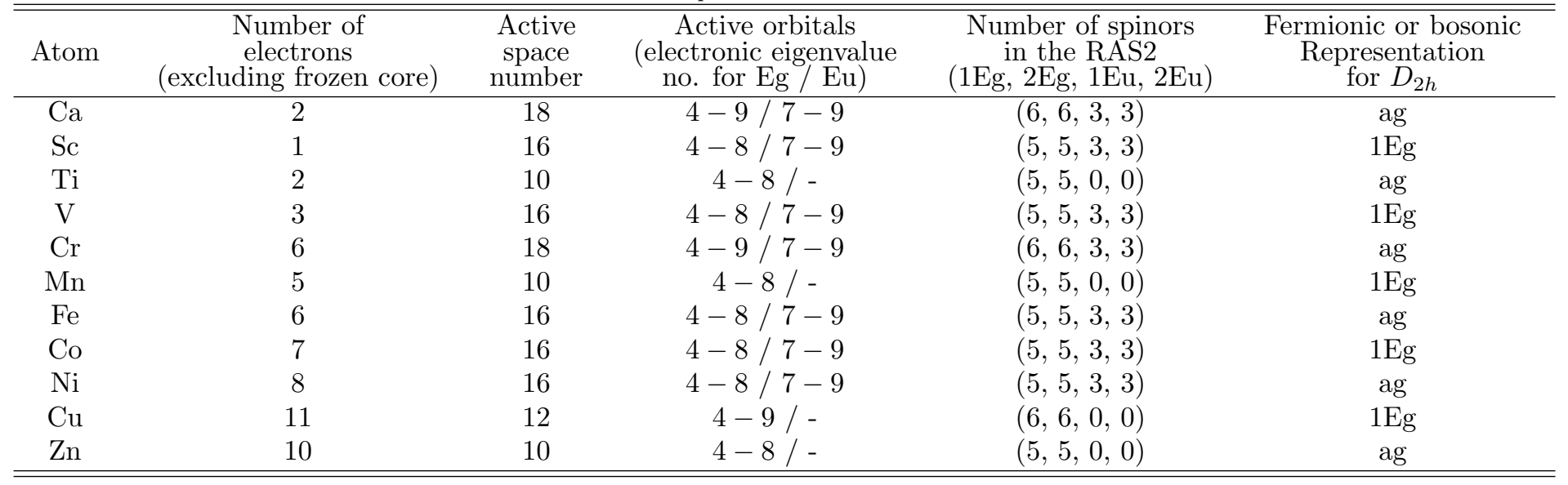

\subsection{Computational details}

We study the spin torque and zeta force of monoatoms. In particular, we take Sc-Zn atoms as examples of transition metals and a $\mathrm{Ca}$ atom as an example of main group elements. As mentioned above, we should derive relativistic four-component wave functions. Hence, our calculations of electron states are carried out by DIRAC10 program package [22]. In our calculations, the Dirac-Coulomb Hamiltonian is used.

For the choices of basis functions, we take the aug-ccpVTZ basis set [23] for the large component of Sc-Zn atoms and the $6-311 \mathrm{G}^{* *}$ basis set [24] for a Ca atom. For the $\mathrm{Ca}$ atom, a different basis set is chosen. However, the accuracy of derived wave functions is comparable with those for other atoms, since the $6-311 \mathrm{G}^{* *}$ basis set has also triple zeta and polarization functions. The contraction of these basis sets is prepared by nonrelativistic calculations, and however, for realizing spin-orbit coupling accurately, the nonrelativistic contraction is not suitable. Hence, all basis sets are decontracted in this work. Basis sets for small components are determined by the unrestricted kinetic balance method.

For Hartree-Fock (HF) calculation, DIRAC10 uses average-of-configuration HF. For some transition metal atoms, both $3 \mathrm{~d}$ and $4 \mathrm{~s}$ shells are open. We specify two open shells appropriately. After the four component HF calculation, configuration interaction (CI) calculations are performed in the restricted active space (RAS) method [25]. The details of CI calculations are summarized in Table 1. In these calculations, we have chosen parameters so that energy levels reported in Ref. [27] are reproduced appropriately. The lowest energy state of each atom is used for the calculation of the spin torque and the zeta force. This calculation is performed by the MRDFT program package [26]. Only active electrons in CI calculations are used for this calculation. In our program package, the vector potential has not been coded. However, the effect of the vector potential by single atom at the steady state is negligibly small.

\section{Result}

\subsection{Spin torque and zeta force}

In this section, we investigate the spin torque and zeta force of monoatoms of transition metals. Before this calculation, we calculate some other low energy states and show their results, in order to check our electronic states. The results of electron states for a Fe atom are shown in Table 2 as an example. These data show that spin-orbit interaction breaks degeneracies and these states show fine structure depending on their total angular momentum, J. Our results realize sufficiently the energy difference between these states. Each $J$ state has its multiplicity $2 J+1$, and the corresponding number of electron states are derived for all values of total angular momentum.

Next, we calculate the spin torque and zeta force of the ground state of monoatoms of transition metals. At the steady state, the expectation value of spin angular momentum density operator has no time variation. Therefore, two terms in the right-hand side of Eq. (2) should cancel out each other. We pick up again a Fe atom as an example and show its spin torque and zeta force distributions in Fig. 1. The first state of $J=4$ in Table 2 is chosen as mentioned above. The directions of the spin torque and zeta force are opposite at every point. As seen in Fig. 1, we can confirm that our calculations realize the cancellation well. Residual values are smaller by at least one order of magnitude, and this difference arises from the deviation between the true wave function and ours, e.g., due to the finite expansion of gaussian basis functions and the use of the Dirac-Coulomb Hamiltonian. As a result, it is shown that a Fe atom has nonzero local spin torque, and the spin steady state is realized by the cancellation between the spin torque and zeta force. We also check the cancellation between the local spin torque and zeta force for other states of a $\mathrm{Fe}$ atom. We calculated the local spin torque and zeta force at $132651(=51 \times 51 \times 51)$ grid points in the cube, whose sides are 2 bohr and center is at a nucleus, and derived the average of the norms of them. These average values of the norms of the spin torque and 


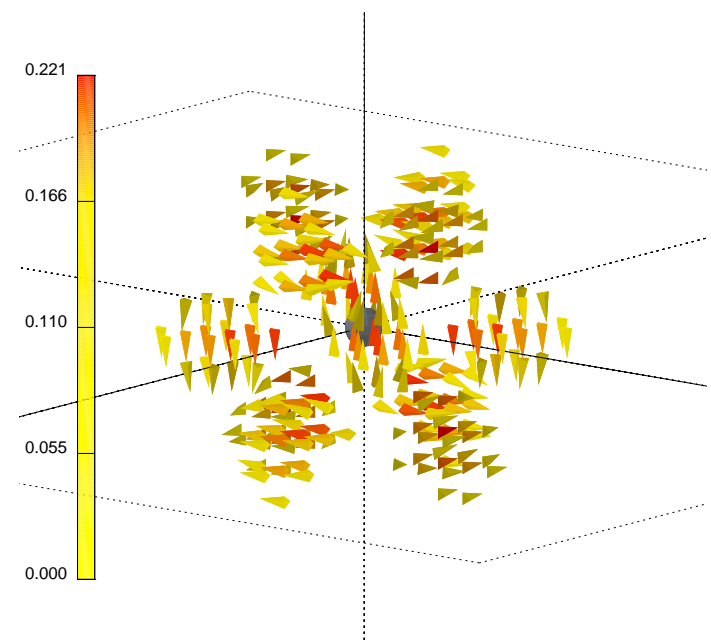

(a)

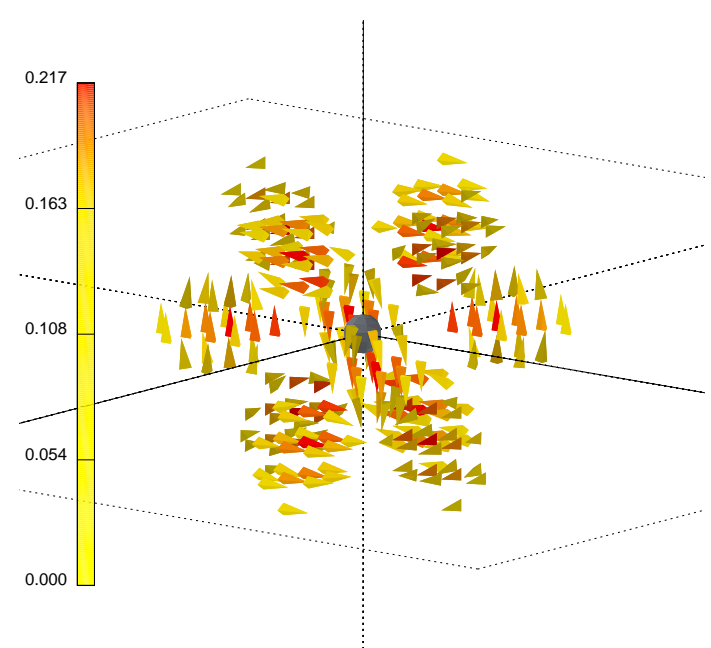

(b)

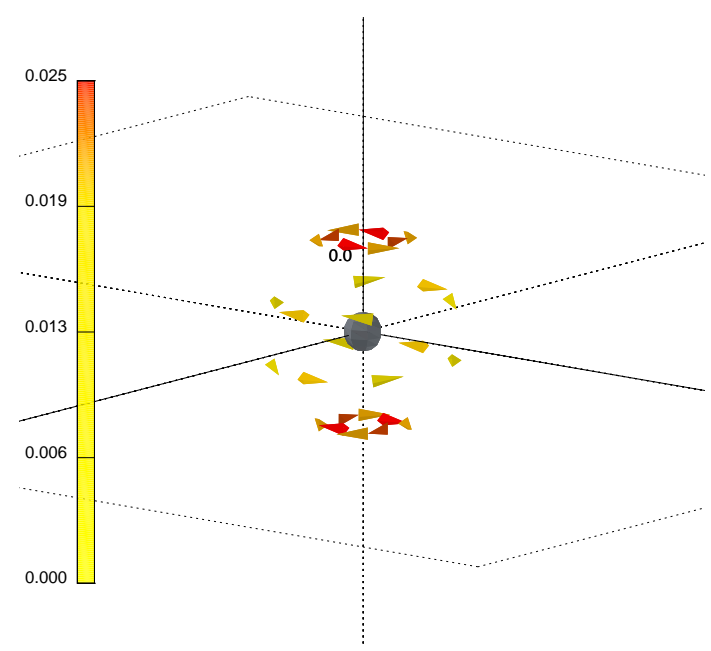

(c)

Figure 1: (Color online) The distributions of the (a) spin torque and (b) zeta force of a Fe atom. Trigonal pyramids represent each vector. Lower threshold values are taken as 0.163 (a.u.) for both panels. (c) Sum of the spin torque and zeta force. The threshold value is 0.019 (a.u.).
Table 2: Energy levels of a Fe atom and the average values of the norm of the spin torque and zeta force. The level of the ground state is zero. The index $J$ denotes the total angular momentum quantum number. Experimental data from Ref. [27] are stated as boldface letters.

\begin{tabular}{|c|c|c|c|c|}
\hline \multicolumn{2}{|c|}{$J$ Irrep } & $\begin{array}{l}\text { Level } \\
\left(\mathrm{cm}^{-1}\right)\end{array}$ & $\begin{array}{l}\text { Avg. of spin torque } \\
\text { (a.u.) }\end{array}$ & $\begin{array}{c}\text { Avg. of zeta force } \\
\text { (a.u.) }\end{array}$ \\
\hline \multirow[t]{10}{*}{4} & & 0.00 & & \\
\hline & & 0.00 & $9.59 \times 10^{-3}$ & $9.60 \times 10^{-3}$ \\
\hline & & 0.00 & $9.05 \times 10^{-3}$ & $9.06 \times 10^{-3}$ \\
\hline & $\mathrm{ag}$ & 0.00 & $3.90 \times 10^{-3}$ & $3.90 \times 10^{-3}$ \\
\hline & & 0.00 & $5.61 \times 10^{-3}$ & $5.61 \times 10^{-3}$ \\
\hline & & 0.00 & $3.29 \times 10^{-2}$ & $3.29 \times 10^{-2}$ \\
\hline & & 0.00 & $8.06 \times 10^{-3}$ & $8.06 \times 10^{-3}$ \\
\hline & & 0.00 & $8.13 \times 10^{-3}$ & $8.13 \times 10^{-3}$ \\
\hline & $\mathrm{bg}$ & 0.00 & $8.59 \times 10^{-2}$ & $8.59 \times 10^{-2}$ \\
\hline & & 0.00 & $2.89 \times 10^{-2}$ & $2.89 \times 10^{-2}$ \\
\hline \multirow[t]{8}{*}{3} & & 415.933 & & \\
\hline & & 455.13 & $8.32 \times 10^{-2}$ & $8.32 \times 10^{-2}$ \\
\hline & $\mathrm{ag}$ & 455.13 & $5.56 \times 10^{-3}$ & $5.56 \times 10^{-3}$ \\
\hline & & 455.13 & $6.63 \times 10^{-3}$ & $6.64 \times 10^{-3}$ \\
\hline & & 455.13 & $5.74 \times 10^{-3}$ & $5.74 \times 10^{-3}$ \\
\hline & & 455.13 & $5.33 \times 10^{-3}$ & $5.33 \times 10^{-3}$ \\
\hline & bg & 455.13 & $1.00 \times 10^{-1}$ & $1.00 \times 10^{-1}$ \\
\hline & & 455.13 & $1.45 \times 10^{-2}$ & $1.45 \times 10^{-2}$ \\
\hline \multirow[t]{6}{*}{2} & & 704.003 & & \\
\hline & & 774.10 & $3.20 \times 10^{-2}$ & $3.20 \times 10^{-2}$ \\
\hline & ag & 774.10 & $3.15 \times 10^{-3}$ & $3.15 \times 10^{-3}$ \\
\hline & & 774.10 & $6.39 \times 10^{-3}$ & $6.40 \times 10^{-3}$ \\
\hline & & 774.10 & $9.27 \times 10^{-2}$ & $9.27 \times 10^{-2}$ \\
\hline & bg & 774.10 & $5.65 \times 10^{-2}$ & $5.65 \times 10^{-2}$ \\
\hline \multirow[t]{4}{*}{1} & & 888.132 & & \\
\hline & $\mathrm{ag}$ & 978.90 & $4.03 \times 10^{-3}$ & $4.03 \times 10^{-3}$ \\
\hline & & 978.90 & $1.15 \times 10^{-2}$ & $1.15 \times 10^{-2}$ \\
\hline & bg & 978.90 & $8.18 \times 10^{-2}$ & $8.18 \times 10^{-2}$ \\
\hline \multirow[t]{2}{*}{0} & & 978.074 & & \\
\hline & $\mathrm{ag}$ & 1079.23 & $4.32 \times 10^{-4}$ & $4.33 \times 10^{-4}$ \\
\hline
\end{tabular}

zeta force are shown in Table 2 as consistency check. If the local spin torque cancels out with the local zeta force, these averages must have the same value. We can see the average values of the spin torque are the same as those of the zeta force. Therefore, this results strongly suggest the cancellation between the local spin torque and zeta force.

This nonzero local spin torque is not specific for the $\mathrm{Fe}$ atom. We confirmed that this can also be seen for other transition metals, Sc-Ni atoms. The average values of the spin torque and total torque for transition metals, Sc-Zn atoms, are shown in Table 3 . The cancellation between the spin torque and zeta force is clearly seen for $\mathrm{Sc}-\mathrm{Ni}$ atoms, the norm of total torque vector is much smaller than that of the spin torque, except for $\mathrm{Cu}$ and $\mathrm{Zn}$ atoms. We can also see that the magnitudes of the spin torque for $\mathrm{Sc}, \mathrm{Cu}$, and $\mathrm{Zn}$ are extremely smaller than those of other transition metals. We discuss the origin of this difference in later part of this section. 
Table 3: The average values of the norm of the spin torque and total torques (spin torque + zeta force) for transition metals. Configuration (Config.) of valence electrons for ground states are also shown. The electrons included in the calculation of the spin torque are stated as boldface letters. The index $J$ denotes the total angular momentum quantum number.

\begin{tabular}{|c|c|c|c|c|c|c|c|c|c|c|}
\hline & Sc & $\overline{\mathrm{Ti}}$ & $\overline{\mathrm{V}}$ & $\mathrm{Cr}$ & $\mathrm{Mn}$ & $\mathrm{Fe}$ & Co & $\mathrm{Ni}$ & $\mathrm{Cu}$ & $\mathrm{Zn}$ \\
\hline $\begin{array}{l}\text { Spin torque } \\
\text { (a.u.) }\end{array}$ & $1.52 \times 10^{-8}$ & $2.62 \times 10^{-2}$ & $2.29 \times 10^{-1}$ & $2.30 \times 10^{-2}$ & $3.24 \times 10^{-2}$ & $9.60 \times 10^{-3}$ & $1.80 \times 10^{-1}$ & $1.09 \times 10^{-2}$ & $1.78 \times 10^{-9}$ & $5.36 \times 10^{-11}$ \\
\hline $\begin{array}{l}\text { Total } \\
\text { (a.u.) }\end{array}$ & $8.98 \times 10^{-12}$ & $3.65 \times 10^{-5}$ & $8.66 \times 10^{-5}$ & $1.21 \times 10^{-5}$ & $3.24 \times 10^{-4}$ & $2.48 \times 10^{-4}$ & $1.28 \times 10^{-4}$ & $2.09 \times 10^{-4}$ & $1.78 \times 10^{-9}$ & $5.38 \times 10^{-11}$ \\
\hline $\begin{array}{c}\text { Config. } \\
J\end{array}$ & $\begin{array}{c}\mathbf{3 d}^{\mathbf{1}} 4 \mathrm{~s}^{2} \\
1^{1 / 2}\end{array}$ & $\begin{array}{c}3 \mathbf{d}^{2} 4 \mathrm{~s}^{2} \\
2\end{array}$ & $\begin{array}{c}3 \mathbf{d}^{3} 4 \mathrm{~s}^{2} \\
1^{1 / 2}\end{array}$ & $\begin{array}{c}3 d^{5} 4 s^{1} \\
3\end{array}$ & $\begin{array}{c}3 \mathbf{d}^{\mathbf{5}} 4 \mathrm{~s}^{2} \\
2^{1} / 2\end{array}$ & $\begin{array}{c}3 \mathbf{d}^{6} 4 \mathrm{~s}^{2} \\
4\end{array}$ & $\begin{array}{c}3 \mathbf{d}^{7} 4 \mathrm{~s}^{2} \\
4^{1} / 2\end{array}$ & $\begin{array}{c}3 \mathrm{~d}^{8} 4 \mathrm{~s}^{2} \\
4\end{array}$ & $\begin{array}{c}3 \mathbf{d}^{\mathbf{1 0}} \mathbf{4} \mathbf{s}^{\mathbf{1}} \\
1 / 2\end{array}$ & $\begin{array}{c}\mathbf{3 d}^{\mathbf{1 0}} 4 \mathrm{~s}^{2} \\
0\end{array}$ \\
\hline
\end{tabular}

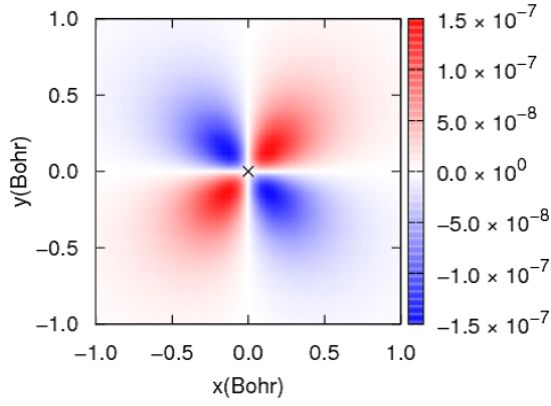

(a)

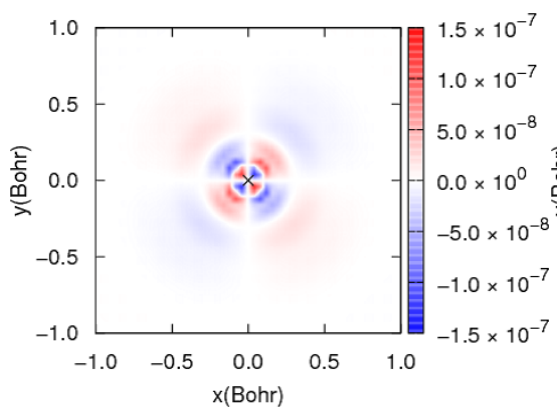

(c)

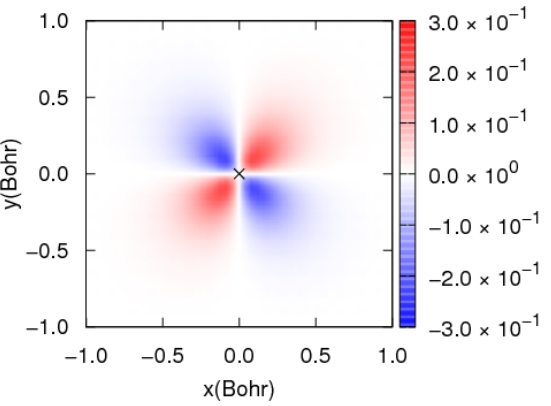

(b)

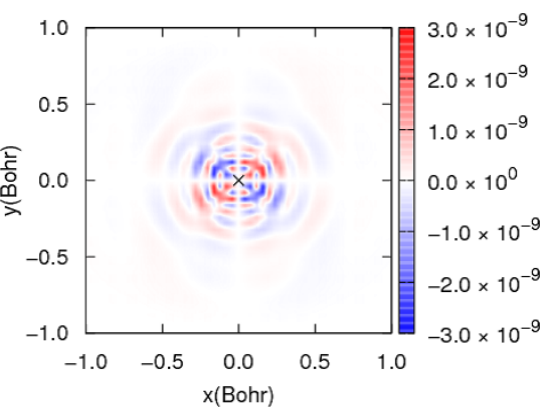

(d)

Figure 2: (Color online) The $z$-component of the spin torque for transition metals on the plane $z=0$. (a) Sc, (b) Fe, (c) Cu, and (d) Zn.

Next, we discuss the distribution patterns of the spin torque of monoatoms of transition metals. We show the distribution pattern for each transition metal on the $z=0$ plane in Fig. 2, as examples. The ground states are used for this calculation, according to the conditions in Table 1. The distribution patterns of the spin torque of Sc and Fe atoms are similar to each other in spite of the difference of the magnitude. We confirmed that this similarity of the distribution pattern is seen for $\mathrm{Sc}-\mathrm{Ni}$ atoms in three dimensional one. Hence, only Sc and Fe atoms are shown among Sc-Ni atoms. On the other hand, distribution patterns for $\mathrm{Cu}$ and $\mathrm{Zn}$ atoms are drastically different from other transition metals and have somewhat noisy patterns. We consider that the smallness of the spin torque and zeta force is beyond the accuracy of our calculation.

$\mathrm{Cu}$ and $\mathrm{Zn}$ atoms are different from other transition metals, since d-orbitals of these atoms are fully occupied. In addition, Sc atom is also different from other transition atoms, since it has only one d-orbital electron. We speculate that the smallness of the spin torque and zeta force of these atoms is due to these specific config- uration of d-orbital electrons. First, to show the importance of the d-orbital electrons on the spin torque and zeta force, we calculated the spin torque and zeta force of main group elements, up to calcium. These atoms showed extremely small spin torque and zeta force of the order of $10^{-10}-10^{-12}$ (a.u.), which are much smaller than those of Sc-Ni atoms. As an example, the distributions of the spin torque and zeta force of a Ca atom are shown in Fig. 3. We can see that the spin torque and zeta force are very small and the cancellation between the spin torque and zeta force is not seen. Hence, we consider that the spin torque and zeta force of this atom are too small for our calculation accuracy to realize the cancellation. Particularly, as can be seen in Eq. (3), the spin torque is defined as the difference between the off-diagonal elements of the relativistic stress tensor density operator. Hence, very high accuracy is required to calculate the spin torque for these main group elements accurately. In fact, the typical order of off-diagonal elements of stress tensor density is $10^{-1}-10^{-3}$ (a.u.). Hence, the accuracy about ten or more order of magnitude is required to derive reliable 


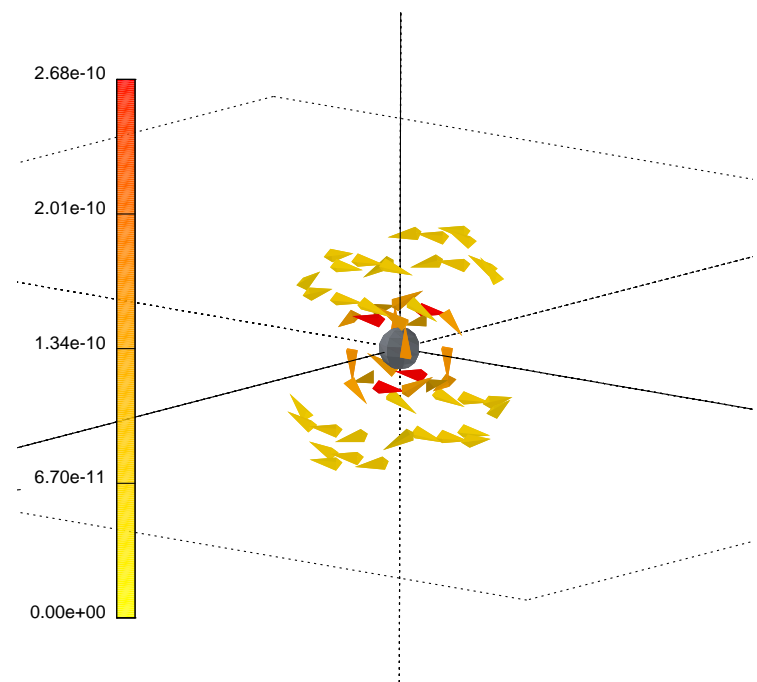

(a)

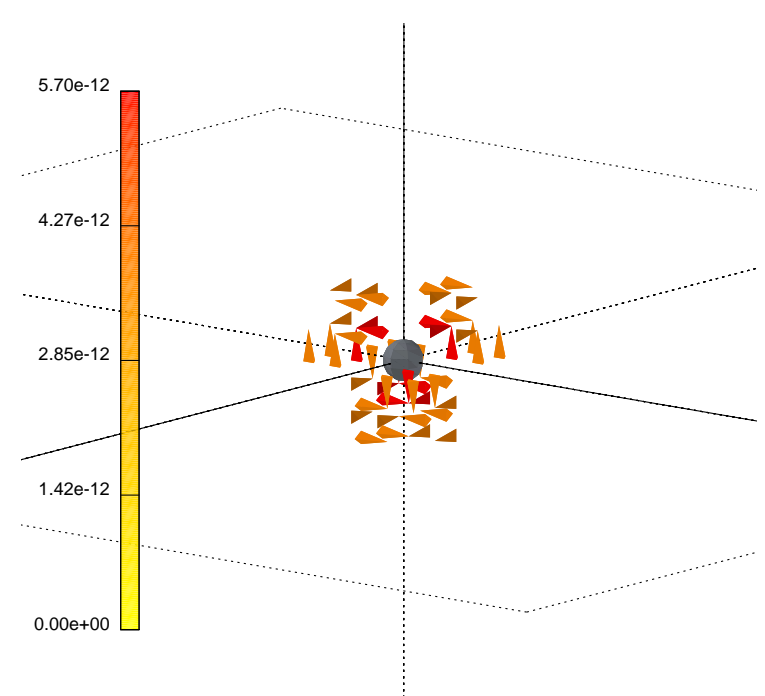

(b)

Figure 3: (Color online) The distributions of the (a) spin torque and (b) zeta force of a $\mathrm{Ca}$ atom. Their lower threshold values are taken as (a) $6.70 \times 10^{-11}$ (a.u.), (b) $2.85 \times 10^{-12}$ (a.u.), respectively.

results of the spin torque. The difference between main group elements up to calcium and transition metals is dorbital electrons: e.g. the ground state of the Ca atom is known as $[\mathrm{Ar}] 4 \mathrm{~s}^{2}$. Hence, we consider that larger torque of transition metals is due to the contribution of d-orbital electrons.

If d-orbital electrons have important effects on the spin torque and zeta force, an excited state of a $\mathrm{Ca}$ atom which has a d-orbital electron, should have much larger spin torque and zeta force compared to the ground state. The fourth excited state of a $\mathrm{Ca}$ atom has [Ar] 4s3d configuration. The spin torque of this excited state is shown in Fig. 4. For this state, the spin torque has much larger value than the ground state as expected. In addition, we confirmed the cancellation between the spin torque and

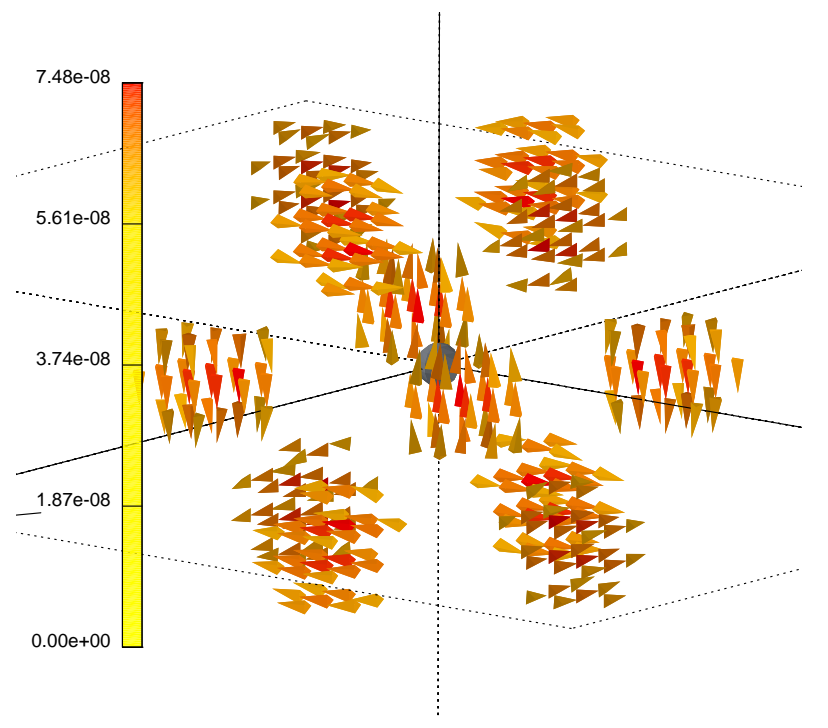

Figure 4: (Color online) The distributions of the spin torque of the excited state $[\mathrm{Ar}] 4 \mathrm{~s} 3 \mathrm{~d}$ for the $\mathrm{Ca}$ atom. The lower threshold value is taken as $6.3 \times 10^{-8}$ (a.u.).

the zeta force. The average value of the norm of the spin torque in this calculation is $1.04 \times 10^{-8}$ (a.u.), which is similar magnitude to that of the Sc atom, which has the same d-orbital electron configuration.

Here, we return to the difference of magnitude of torques between transition metals. As shown in Table 3, all of their torques, except for $\mathrm{Cu}$ and $\mathrm{Zn}$ atoms, are canceled and the distribution patterns are the same. However, the values of the torques of the Sc atom is much smaller than that of Ti-Ni atoms as mentioned above. The Sc atom has only one electron in valence d-orbitals. The fourth excited state of a Ca atom has also the same d-orbital configuration and has similar magnitude to that of the Sc atom. Therefore, it is supposed that the interaction between electrons in dorbitals makes spin torque and zeta force large as $\mathrm{Ti}-\mathrm{Ni}$ atoms. Then, we consider the second excited state of the Sc atom, $[\mathrm{Ar}] 3 \mathrm{~d}^{2} 4 \mathrm{~s}$, and calculate its spin torque and zeta force in order to test our consideration, as the Ca atom above. The result is shown in Fig. 5. It is found that the magnitude of the spin torque of the excited state is significantly larger than that of the ground state, and it has roughly the same order of the torque of $\mathrm{Ti}-\mathrm{Ni}$ atoms. This result reveals that d-orbital electrons dominantly contribute to the spin torque and zeta force and more than one d-orbital electron makes torques larger.

For $\mathrm{Cu}$ and $\mathrm{Zn}$ atoms, the spin torque and zeta force are much smaller than other transition metals. The cancellation between the spin torque and zeta force cannot be seen, since it is beyond the accuracy of our calculation. From these results, we consider that the spin torque and zeta force have only small value for fully occupied dorbitals. Therefore, we calculate the second excited state $[\mathrm{Ar}] 3 \mathrm{~d}^{9} 4 \mathrm{~s}^{2}$ of the $\mathrm{Cu}$ atom. The spin torque in this ex- 


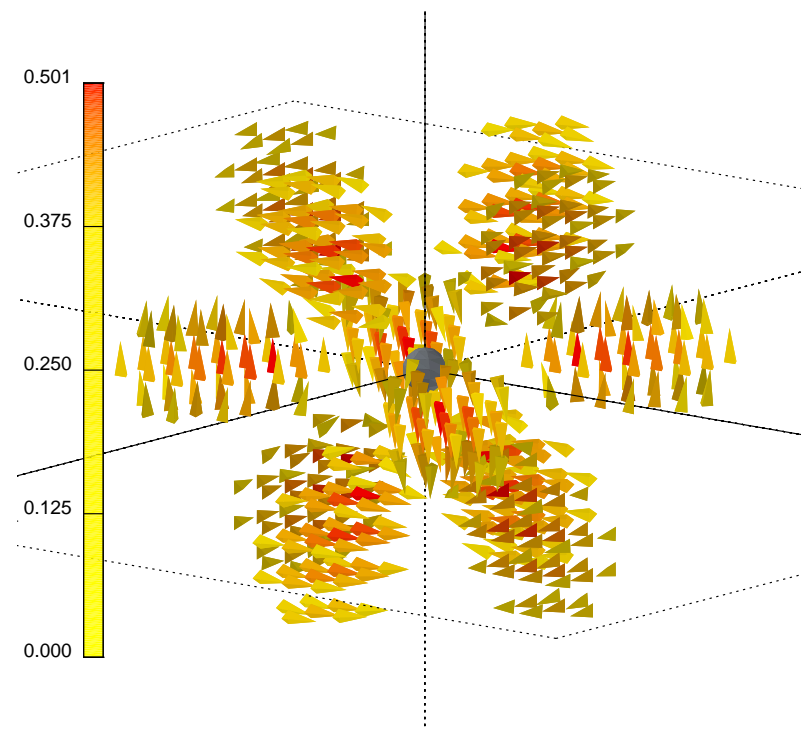

Figure 5: (Color online) The distributions of the spin torque of the $\mathrm{Sc}$ atom for the excited state $[\mathrm{Ar}] 3 \mathrm{~d}^{2} 4 \mathrm{~s}$. The lower threshold value is taken as 0.375 (a.u.).

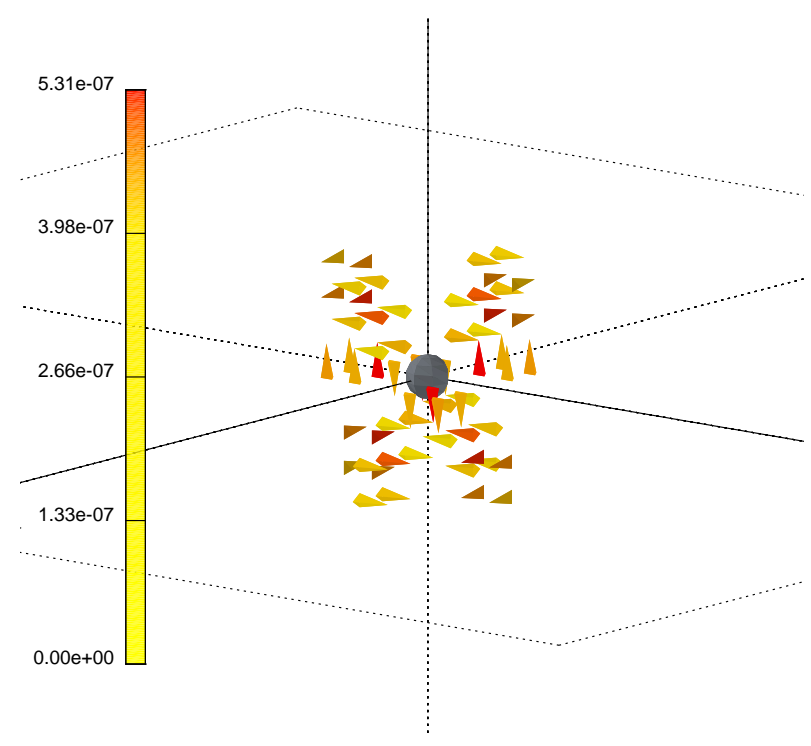

Figure 6: (Color online) The distributions of the spin torque of the excited state $[\mathrm{Ar}] 3 \mathrm{~d}^{9} 4 \mathrm{~s}^{2}$ for the $\mathrm{Cu}$ atom. The lower threshold value is taken as $3.98 \times 10^{-7}$ (a.u.).

cited state is shown in Fig. 6. The spin torque and zeta force have the same distribution pattern as that of other transition metals. The cancellation between them was also confirmed in this excited state. The average value of the norm of the spin torque in this calculation is $1.22 \times 10^{-8}$ (a.u.), which is the same order of the torque of a Ca atom in the excited state and a Sc atom in the ground state. In $\mathrm{Cu}$ and $\mathrm{Zn}$ atoms, d-orbitals are fully occupied in the ground state, and contributions from d-orbital electrons to the spin torque and zeta force seem to be suppressed. For the excited state of the $\mathrm{Cu}$ atom, the number of occupied d-orbitals are nine, and its torques are as large as those of states whose d-orbitals are occupied by only one electron. As a result, it can be concluded that the spin torque and zeta force are significantly small for fully occupied dorbitals.

The spin torque and zeta force are expected to be closely related to the spin-orbit interaction. In a future work, we will study the relation between the spin torque and the spin-orbit interaction.

\subsection{Chirality density}

We analyze the local chirality density, defined as Eq. (10), which is the potential for zeta force. Although the expectation value of the zeta force is zero for monoatoms, the local chirality density can exist in them. The chirality and electron density distributions of ferromagnetic metals, Fe, Co, and $\mathrm{Ni}$ are shown in Fig. 7. Green (light) and blue (dark) envelopes represent positive and negative chirality density iso-surface, respectively. For chirality density, these three atoms have closely similar distribution patterns to each other. The positive and negative regions are inverted for the $\mathrm{Ni}$ atom. However, since the system has rotational symmetry, this is considered to be the same pattern. This similarity of the patterns is an expected result, since the distribution patterns of the zeta force are the same. (Note that the zeta force is proportional to the gradient of the chirality density.) We checked that the distribution patterns of Sc-Mn atoms are also the same. As discussed in Sec. 2.1, the nonzero chirality density is dissipated by the electron mass term. However, surprisingly, the nonzero local chirality density exists even in steady states and make the zeta force probably due to spin-orbit interaction. In addition, we compare the distributions of the chirality density and the electron density. Pink (transparent) surface represents electron density. The distributions of the electron density are much different from each other. The reason of the difference of the electron density is that occupied d-orbitals are different for these atoms. Hence, it is very interesting that the chirality density has the common distribution pattern though the distribution of the electron density is significantly different. This is explained by the distribution of the small components of four-component wave function. As mentioned in Sec. 2.1, the chirality density (zeta force) is derived as the product of large and small components of a wave function. The electron density is dominantly determined by the large component. Hence, the difference of the large component is compensated by the small component. Actually, we have checked that the distributions of the small component of wave function are much different between these atoms. This result is the unique feature of the spin, which can never be explained in the nonrelativistic property of electrons. 


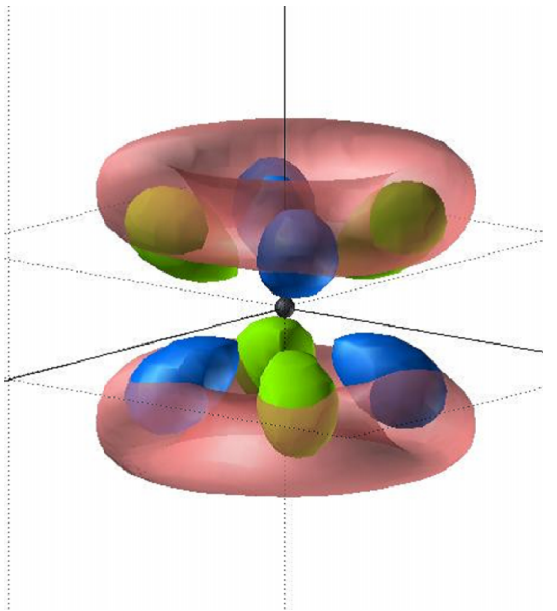

(a)

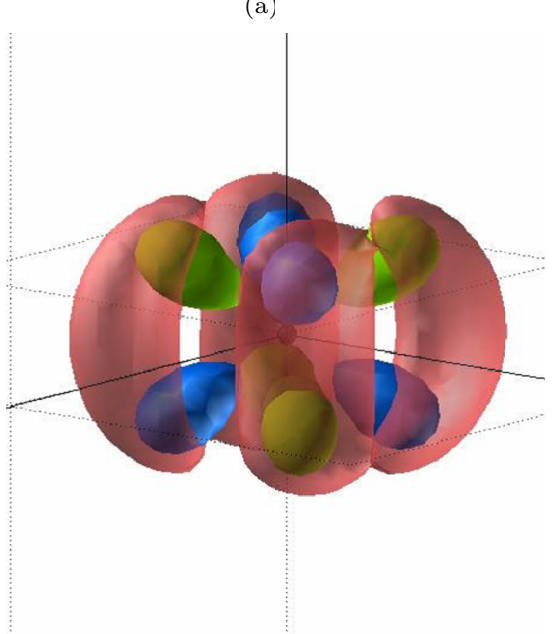

(b)

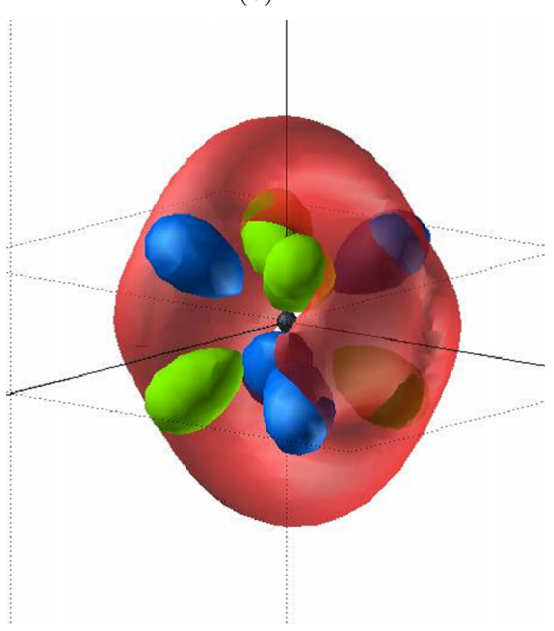

(c)

Figure 7: (Color online) The iso-surfaces of the chirality density and electron density of (a) Fe, (b) Co and (c) Ni atoms. Green (light) and blue (dark) envelopes represent positive and negative chirality density iso-surface, respectively. Pink (transparent) surface represents electron density of active electrons in our CI calculations. Each iso-surface value of chirality/electron density is taken as (a): $\pm 2.30 \times 10^{-2} / 1.44,(\mathrm{~b}): 4.77 \times 10^{-1} / 1.83,(\mathrm{c}): \pm 3.23 \times 10^{-2} / 2.72$, respectively. The unit of all panels is atomic unit.

\section{Conclusion}

In this study, we have analyzed the spin torque and zeta force of transition metals. We have shown that transition metals which have partially occupied d-orbitals, have large local spin torque. This nonzero local torque are canceled out by the zeta force, and hence the observed spin steady state is realized in spite of the existence of nonzero spin torque. On the other hand, transition metals which have fully occupied d-orbitals, and main group elements up to calcium, which have no occupied d-orbital, have almost no local spin torque. We have carefully confirmed that large spin torque and zeta force are attributed to d-orbital electrons, using excited states of $\mathrm{Ca}, \mathrm{Sc}$, and $\mathrm{Cu}$ atoms.

In addition, we have surprisingly found the existence of local chirality density in atoms even at the steady state, though the Dirac mass term of the electron has effects to wash out nonzero chirality density. Although the distribution patterns of electron density are drastically different between transition metal monoatoms, because of different occupied orbitals, those of the chirality density have the common pattern for transition metals from Sc to Ni atoms. It is explained due to the compensation by the distribution of small components of wave functions for the different distribution of large components.

\section{Acknowledgements}

This work was partially supported by Grant-in-Aid from the Japan Society of the promotion of science (22550011).

\section{References}

[1] S. Wolf, D. Awschalom, R. Buhrman, J. Daughton, S. Von Molnár, M. Roukes, A. Chtchelkanova, and D. Treger, Science 294, 1488 (2001).

[2] S. Maekawa and T. Shinjō, Spin dependent transport in magnetic nanostructures (CRC, 2002).

[3] J. Slonczewski, J. Magn. Magn. Mater. 159, L1 (1996).

[4] L. Berger, Phys. Rev. B 54, 9353 (1996).

[5] H. Kohno, G. Tatara, and J. Shibata, J. Phys. Soc. Japan 75, 3706 (2006).

[6] S. Zhang and Z. Li, Phys. Rev. Lett. 93, 127204 (2004).

[7] A. Thiaville, Y. Nakatani, J. Miltat, and Y. Suzuki, Europhys. Lett. 69, 990 (2005).

[8] S. Barnes and S. Maekawa, Phys. Rev. Lett. 95, 107204 (2005).

[9] A. Tachibana, J. Mol. Model. 11, 301 (2005).

[10] A. Tachibana, J. Mol. Struct.: THEOCHEM 943, 138 (2010).

[11] M. Senami, J. Nishikawa, T. Hara, and A. Tachibana, J. Phys. Soc. Japan 79, 084302 (2010).

[12] A. Tachibana, Theor. Chem. Accou. 102, 188 (1999).

[13] A. Tachibana, J. Chem. Phys. 115, 3497 (2001).

[14] A. Tachibana, in AIP Conf. Proc. (2002), vol. 612, p. 105.

[15] A. Tachibana, Int. J. Quantum Chem. 100, 981 (2004).

[16] A. Tachibana and R. Parr, Int. J. Quantum Chem. 41, 527 (1992).

[17] A. Tachibana, Int. J. Quantum Chem. 32, 181 (1987).

[18] P. Szarek, Y. Sueda, and A. Tachibana, J. Chem. Phys. 129, 094102 (2008).

[19] A. Tachibana, Int. J. Quantum Chem. 57, 423 (1996).

[20] A. Tachibana, K. Nakamura, K. Sakata, and T. Morisaki, Int. J. Quantum Chem. 74, 669 (1999). 
[21] R. A. Hegstrom, J. P. Chamberlain, K. Seto, and R. G. Watson, Am. J. Phys., 56 (1988) 1086; R. A. Hegstrom, J. Mol. Struct.:(THEOCHEM), 232 (1991) 17; R. Bast, A. Koers, A. S. P. Gomes, M. Iliaš, L. Visscher, P. Schwerdtfeger, and T. Saue, Phys. Chem. Chem. Phys., 13 (2011) 864.

[22] DIRAC, a relativistic ab initio electronic structure program, Release DIRAC10 (2010), written by T. Saue, L. Visscher and H. J. Aa. Jensen, with contributions from R. Bast, K. G. Dyall, U. Ekström, E. Eliav, T. Enevoldsen, T. Fleig, A. S. P. Gomes, J. Henriksson, M. Iliaš, Ch. R. Jacob, S. Knecht, H. S. Nataraj, P. Norman, J. Olsen, M. Pernpointner, K. Ruud, B. Schimmelpfennnig, J. Sikkema, A. Thorvaldsen, J. Thyssen, S. Villaume, and S. Yamamoto (see http://dirac.chem.vu.nl).

[23] N. Balabanov and K. Peterson, J. Chem. Phys. 123, 064107 (2005)

[24] J. Blaudeau, M. McGrath, L. Curtiss, and L. Radom, J. Chem. Phys. 107, 5016 (1997)

[25] L. Visscher, O. Visser, P. Aerts, H. Merenga, and W. Nieuwpoort, Comp. Phys. Comm. 81, 120 (1994).

[26] M. Senami, K. Ichikawa, P. Szarek, K. Doi, K. Nakamura, A. Tachibana, Molecular Regional DFT Program Package, ver. 3, Tachibana Lab., Kyoto Univ. (2008).

[27] C. Moore, Bur. Stand. Circ 467 (1952). 